\title{
Intencionalidade em Tomasello, Searle, Dennett e em Abordagens Comportamentais da Cognição Humana ${ }^{1}$
}

\author{
Sylvio Állan ${ }^{2}$ \\ Carlos Barbosa Alves de Souza \\ Universidade Federal do Pará
}

\begin{abstract}
RESUMO - A abordagem de Tomasello da evolução da cognição humana busca integrar processos biológicos, comportamentais e culturais em um mesmo sistema explicativo. No entanto, uma das principais críticas a essa abordagem é a necessidade de uma melhor elaboração do conceito de intencionalidade. O objetivo do presente trabalho foi: (1) analisar o tratamento de Tomasello do conceito de intencionalidade; e (2) estabelecer interlocuções desse tratamento com teorias da intencionalidade na filosofia da mente e com abordagens funcionalistas da cognição humana na psicologia comportamental. Sugerimos que o tratamento do conceito de intencionalidade na abordagem de Tomasello é compatível com essas teorias e abordagens. Além disso, a abordagem de Tomasello pode ampliar a investigação de processos simbólicos mais complexos do que aqueles tradicionalmente investigados pela psicologia comportamental.
\end{abstract}

Palavras-Chave: intencionalidade; cognição humana; Tomasello; filosofia da mente; psicologia comportamental.

\section{Intentionality in Tomasello, Searle, Dennett and Behavioral Approaches to Human Cognition}

\begin{abstract}
The approach of Tomasello to the evolution of human cognition attempts to integrate biological, behavioral, and cultural processes within a sole explicative system. A fundamental critic towards it has been the need of a better elaboration of the concept of intentionality. The present paper aimed to: (1) analyze Tomasello's use of the concept of intentionality, and (2) establish dialogues between this use and theories of intentionality in the philosophy of mind and functionalist approaches to human cognition in behavior psychology. The results of this paper indicate that Tomasello's use of the concept of intentionality is compatible to these theories and approaches. Morevover, Tomasello's approach may extend the investigation of more complex symbolic processes than the ones traditionally investigated by behavior psychology.
\end{abstract}

Keywords: intentionality; human cognition; Tomasello; philosophy of mind; behavior psychology.

Em uma revisão teórica da abordagem da evolução da cognição humana, desenvolvida por Tomasello (Tomasello, 1999; Tomasello, Carpenter, Call, Behne \& Moll, 2005a,b), Állan e Souza (2009; Állan, 2007) sugeriram que essa abordagem fornece uma interpretação alternativa para o desenvolvimento cognitivo e simbólico humano, ao integrar processos biológicos, comportamentais e culturais em um mesmo sistema explicativo.

Segundo esses autores, a abordagem de Tomasello possibilita a investigação de repertórios simbólicos mais complexos (p.ex., metacognição, auto-regulação, teoria da mente, redescrição comportamental; ver Tomasello, 1999) do que aqueles tradicionalmente investigados pelas abordagens funcionalistas da cognição humana e da linguagem verbal (p.ex., psicologia comportamental; ver Állan, 2007). Ao mesmo tempo, essa abordagem propõe uma concepção

1 O presente trabalho é derivado da dissertação de mestrado do primeiro autor, sob orientação do segundo autor, apresentada ao Programa de Pós-Graduação em Teoria e Pesquisa do Comportamento, da Universidade Federal do Pará, em 2007, com apoio do Conselho Nacional de Desenvolvimento Científico e Tecnológico (CNPq), por meio de bolsa de estudo.

2 Endereço para correspondência: Rua Manoel Barata, 106, apt. 202, Cruzeiro (Icoaraci). Belém, PA. CEP: 66.810-100.E-mail: sylvioallan@ yahoo.com.br; carlos.souza@pesquisador.cnpq.br de linguagem verbal, como produto de processos cognitivo-funcionais, que permite o estudo de uma variedade de repertórios verbais complexos (p.ex., gramaticalização, repertório narrativo; ver Tomasello, 2003).

Em resumo, Tomasello (1999, 2003; Tomasello et al., $2005 \mathrm{a}, \mathrm{b}$ ) propôs que a cognição humana propriamente dita é um produto evolutivo, derivado de três condições.

A primeira, relativa a adaptações biológicas comuns a outras espécies animais; principalmente, habilidades de compreensão da ação intencional de co-específicos, as quais teriam possibilitado que a espécie humana desenvolvesse uma compreensão dos aspectos causais mediadores das relações físicas entre eventos naturais, assim como, uma compreensão dos aspectos intencionais mediadores das relações sociais entre co-específicos.

A segunda, uma motivação exclusivamente humana para compartilhar intencionalidade com co-específicos, o que teria favorecido o estabelecimento de práticas de colaboração. E a terceira, um mecanismo de evolução cultural, comum a maioria das espécies animais, mas que, especificamente no caso da espécie humana, teria favorecido a acumulação de conhecimentos já adquiridos, em um menor período de tempo e a transmissão desses conhecimentos para gerações posteriores.

O desenvolvimento progressivo dessas três condições ao longo do primeiro ano de vida do indivíduo humano 
culmina por volta dos seus 14 meses de vida, quando ele se torna competente para interagir em práticas culturais com outros indivíduos humanos, simbolicamente competentes; além de adquirir e compartilhar conhecimentos por meio de atividades simbólicas. A participação do indivíduo nessas atividades de colaboração altera profundamente suas habilidades cognitivas já existentes, tornando-as mais complexas (p.ex., metacognição, auto-regulação e re-descrições comportamentais) e possibilitando novas formas de interação cultural - envolvendo ou não a mediação da linguagem verbal - que, por sua vez, retroagem sobre as habilidades cognitivas, e assim por diante.

No entanto, essa abordagem da evolução da cognição humana tem recebido algumas críticas, principalmente em relação à ênfase dada por Tomasello à compreensão e ao compartilhamento da intencionalidade como eventos críticos para a evolução e o desenvolvimento cognitivo humano. Especificamente, essas críticas têm sugerido à necessidade de uma melhor elaboração do conceito de intencionalidade (e outros conceitos relacionados a esse) no contexto da abordagem de Tomasello da evolução da cognição humana, considerando-se as implicações da utilização desse conceito para um retorno às abordagens mentalistas da cognição humana e da linguagem verbal (Állan, 2007; Állan \& Souza, 2009).

Além disso, algumas evidências empíricas têm questionado alguns dados apresentados por Tomasello como suporte às suas hipóteses do desenvolvimento da compreensão e do compartilhamento de intencionalidade durante os primeiros anos de vida da criança (ver Állan, 2007; Állan \& Souza, 2009).

Considerando isso, o presente trabalho pretende analisar o tratamento do conceito de intencionalidade (e de conceitos relacionados a esse), no contexto da abordagem de Tomasello acerca da evolução da cognição humana, buscando identificar possíveis inconsistências no modo como esses conceitos têm sido utilizados nessa abordagem. Buscaremos também estabelecer interlocuções dessa abordagem com outras disciplinas que têm investigado processos cognitivo-lingüísticos. Especificamente, analisaremos o tratamento que o conceito de intencionalidade tem recebido nos trabalhos dos filósofos da mente John R. Searle e Daniel C. Dennett - que têm desenvolvido trabalhos significativos sobre intencionalidade - e compararemos o tratamento desses autores com nossa análise do tratamento do conceito de intencionalidade na abordagem de Tomasello.

Finalmente, na medida em que o tratamento do conceito de intencionalidade na abordagem de Tomasello pode sugerir uma aproximação de pressupostos mentalistas, analisaremos como o conceito de intencionalidade tem sido tratado em algumas abordagens funcionalistas da cognição humana (p.ex., na psicologia comportamental) - e se os tratamentos desse conceito, tanto nessas abordagens quanto na abordagem de Tomasello, são compatíveis.

\section{Intencionalidade}

Embora o conceito de intencionalidade não tenha sido explicitamente definido nos principais trabalhos de Tomasello (1999, 2003; Tomasello \& Cols., 2005a,b), é possível sugerir, a partir da leitura desses trabalhos, que a intencionalidade é caracterizada como uma propriedade de certas habilidades cognitivas de algumas espécies animais de serem "direcionadas para certos objetivos".

Essa interpretação é possível a partir de alguns conceitos que Tomasello apresentou nesses trabalhos. Por exemplo, a ação intencional é caracterizada por ele como aquela "direcionada para" objetivos; a intenção é o planejamento de ações "direcionadas para" objetivos; agentes intencionais são organismos animais que agem no mundo "direcionados para" objetivos; a atenção é a percepção "direcionada para" objetivos (objetos ou estados de coisas do mundo); e a intencionalidade compartilhada são interações colaborativas entre indivíduos humanos "direcionadas para" os mesmos objetivos (Tomasello et al., 2005a).

Enquanto uma característica de diversas espécies animais, a intencionalidade estaria relacionada ao tipo de cognição especifica de cada espécie. Por exemplo, as habilidades cognitivas dos mamíferos e, principalmente, dos primatas em geral, seriam ainda mais sofisticadas e complexas do que as habilidades cognitivas das demais espécies animais; por conseguinte, a intencionalidade daquelas espécies seria também mais complexa.

No caso da espécie humana, sua intencionalidade teria se diferenciado da intencionalidade das demais espécies animais (incluindo, dos mamíferos e dos primatas em geral) devido a uma motivação exclusivamente humana para compartilhar intencionalidade com co-específicos (Tomasello et al., $2005 a, b)^{3}$. Isso teria favorecido o surgimento de atividades de colaboração e, conseqüentemente, o desenvolvimento de uma tradição cultural humana propriamente dita, caracterizada: (1) pelo acúmulo de conhecimentos adquiridos e modificados ao longo do tempo; e (2) pela transmissão desses conhecimentos para gerações posteriores (Tomasello, 1999). Esses conhecimentos teriam também favorecido a aquisição de repertórios verbais (Tomasello, 2003) e modificado significativamente a cognição humana, tornando-a cada vez mais sofisticada e complexa.

O conceito de intencionalidade, aplicado no contexto da abordagem de Tomasello da evolução da cognição humana, destaca a interação dos níveis filogenético, ontogenético e cultural, na medida em que a intencionalidade humana representa o produto de: (1) processos evolutivos biológicos, comuns a outras espécies animais, que atuam no indivíduo humano a partir do seu primeiro ano de vida; e (2) processos evolutivos culturais, exclusivos à espécie humana, que atuam no indivíduo humano a partir do seu segundo ano de vida, quando esse indivíduo começa a interagir com outros indivíduos humanos, simbolicamente competentes, envolvendo o compartilhamento de intencionalidade.

Se for correta nossa interpretação do conceito de intencionalidade na abordagem de Tomasello, podemos sugerir que esse conceito descreve uma propriedade funcional das interações dos organismos animais com seu ambiente, enfatizando aspectos pragmáticos dessas interações (p.ex., objetivos, aquisição de repertórios baseado no uso), sem a necessidade de recorrer a entidades imateriais causal-

3 Para Tomasello et al. (2005a) é justamente a falta dessa habilidade para compartilhar intencionalidade a responsável pelas dificuldades de interação social observadas nos indivíduos humanos autistas. 
mente relevantes (p.ex., representações mentais, crenças, desejos), como proposto pelas abordagens mentalistas da cognição humana. Conseqüentemente, é possível também sugerir que o tratamento do conceito de intencionalidade na abordagem de Tomasello é compatível com uma perspectiva funcionalista da cognição humana (p.ex., psicologia comportamental).

\section{Intencionalidade e Conceitos Afins}

Dois conceitos tradicionalmente relacionados ao conceito de intencionalidade e freqüentemente tratados como sinônimos desse são intenção e intencional. Embora Tomasello não tenha explicitamente proposto uma distinção entre esses três conceitos em seus principais trabalhos, é possível considerar que ele os considere aspectos distintos.

Como já dito anteriormente, Tomasello et al. (2005a) caracterizam a intenção como uma habilidade cognitiva humana, envolvendo o planejamento consciente de uma ação ou conjunto de ações orientadas para objetivos. Na medida em que essa habilidade é também orientada para objetivos, ela pode ser considerada uma habilidade intencional. Quer dizer, a intenção tem na intencionalidade sua propriedade constitutiva, mas nem a intencionalidade se restringe à intenção, nem é sinônimo de intenção.

Especificamente, Tomasello et al. (2005a) propuseram que a intenção é desenvolvida a partir de um sistema de referências "internalizadas" do mundo, constituído por: (1) representações cognitivas da condição atual da realidade; (2) objetivos "esperados"; (3) ações necessárias para atingir esses objetivos; (4) conhecimentos e habilidades já adquiridos; e, (5) estados emocionais relacionados à obtenção ou não desses objetivos.

A complexidade da intenção depende da quantidade e complexidade de elementos que compõem esse sistema de referências. Inclusive, alguns dos elementos que compõem esse sistema podem ser perspectivas de outros indivíduos humanos, quando esses participam do contexto no qual a ação planejada se desenvolve. A vantagem desse tipo de "teoria da simulação", envolvendo a adoção de múltiplas perspectivas, é a de facilitar a antecipação de reações emocionais e comportamentais de outros indivíduos.

Um aspecto interessante no tratamento do conceito de intenção na abordagem de Tomasello é que ele inverte a hipótese de que o planejamento de ações (p.ex., resolução de problemas) é produto de competências simbólicas. Em contrapartida, Tomasello et al. (2005a) propuseram que o planejamento de ações (intenção) e a compreensão de si mesmo e de co-específicos como agentes "planejadores" de ações são habilidades cognitivas pré-requisitos para o surgimento de competências simbólicas.

No entanto, um aspecto problemático com esse tratamento do conceito de intenção é que a suposição de que os indivíduos humanos planejam suas ações construindo "modelos mentais" do mundo torna esse conceito compatível com pressupostos mentalistas da cognição humana. Um desafio seria, por exemplo, tentar conciliar os conceitos de intenção, sistema de referências do mundo e adoção de múltiplas perspectivas, no contexto de uma proposta funcionalista da cognição humana, sem o recurso a qualquer tipo de construto representacionalista.

Em relação ao conceito de intencional - freqüentemente utilizado como sinônimo de "planejado" e como uma qualidade (adjetivo) da intenção (substantivo) - podemos interpretá-lo, por outro lado, como sinônimo de "direcionado para objetivos" e, portanto, como qualidade (adjetivo) da intencionalidade. Uma vez que algumas habilidades cognitivas são caracterizadas pela propriedade de "serem orientadas para objetivos", essas habilidades são consideradas intencionais.

$\mathrm{O}$ conceito de objetivo parece ser o mais ambíguo dentre aqueles propostos por Tomasello, a serem analisados nesse trabalho. Em trabalhos anteriores (Tomasello, 1999, 2003), Tomasello havia destacado aspectos pragmáticos ao caracterizar o conceito de objetivo, embora esse conceito ainda não tivesse sido explicitamente apresentado até aquele momento. Especificamente, o conceito de objetivo podia ser interpretado como uma condição da realidade capaz de afetar a atividade dos organismos. Em trabalhos mais recentes (Tomasello et al., 2005a,b), Tomasello passou a adotar uma postura representacionalista ao caracterizar alguns dos seus conceitos na abordagem da evolução da cognição humana e, dentre eles, o conceito de objetivo.

Segundo Tomasello et al. (2005a), o objetivo se refere a uma condição "desejada" da realidade, não a sua condição "atual”. A realidade desejada torna-se a realidade atual por meio da ação intencional, a qual envolve uma intenção (planejamento da ação) e a realização de uma ação propriamente dita. Considere, por exemplo, um indivíduo que "deseja" abrir uma porta. A porta fechada é a condição atual da realidade. A porta aberta é a condição da realidade desejada pelo indivíduo (objetivo). O indivíduo é capaz de agir intencionalmente para abrir a porta porque ele é capaz de planejar seu curso de ação (p.ex., caminhando em direção à porta, solicitando a alguém que abra a porta), formando uma representação cognitiva da porta aberta, antes mesmo de se engajar na atividade que produzirá (ou não) o objetivo desejado.

Assim como no caso do conceito de intenção, o conceito de objetivo pode ser problemático e incompatível com uma interpretação funcionalista, devido à hipótese de representações cognitivas de objetos e estados de coisas do mundo. Além disso, sugerimos que esse conceito demanda uma elaboração mais precisa, visto que sua definição alterna entre aspectos funcionais e representacionalistas.

Finalmente, em relação ao conceito de motivação para compartilhar_intencionalidade (Tomasello et al., 2005a,b) um dos conceitos mais centrais na abordagem de Tomasello - pouco esclarecimento é fornecido quanto à natureza dessa motivação. Se ela é um produto biológico, a partir de quais adaptações biológicas ela teria se desenvolvido na espécie humana? Se ela é um produto cultural, como ela teria se desenvolvido no primeiro ano de vida do indivíduo humano, mas, antes do estabelecimento das primeiras interações culturais desse indivíduo? Se ela é um produto ontogenético, porque ela não se desenvolve nos indivíduos autistas?

Uma alternativa para essa questão é interpretar a motivação para compartilhar intencionalidade como um fenômeno disposicional (Ryle, 1949/1969), i.e., como padrões ou tendências de ocorrências de interações humanas, caracterizados 
pelo "compartilhamento" de objetivos com co-específicos. Compartilhar, nesse sentido, significa que dois ou mais indivíduos colaboram entre si para atingir um mesmo objetivo.

Assim, não faria sentido perguntar: "qual a natureza da motivação para compartilhar intencionalidade?", porque a motivação, enquanto um fenômeno disposicional, não é um fato ontológico. O que devemos de fato perguntar é: "por quais processos a espécie humana teria desenvolvido formas de coordenar estratégias de colaboração para atingir objetivos comuns?". E a resposta para essa questão necessita da interpretação de como a interação dos níveis filogenético, ontogenético e cultural poderia ter levado a espécie humana a se comportar desse modo.

\section{John R. Searle e a Teoria Naturalista da Intencionalidade}

Searle $(1983,1990 a, b, 1992,1997,1998,2004)$ desenvolveu uma abordagem naturalista-biológica da cognição humana que busca analisar o modo de funcionamento da mente em termos de configuração sistêmica do cérebro. Segundo ele, a mente é um modo de organização particular do cérebro, caracterizada por uma capacidade de operar em nível "consciente", e por uma propriedade reguladora das interações humanas com o mundo, denominada Intencionalidade (com "I" maiúsculo). Um estado intencional seria, de acordo com Searle, qualquer estado ou processo mental que possui essa propriedade.

$\mathrm{O}$ tratamento de Searle do conceito de Intencionalidade enfatiza relações semânticas ("aboutness") entre estados intencionais e objetos ou estados de coisas do mundo. Podemos identificar nessas relações: (1) um conteúdo intencional específico, que determina as condições de satisfação do estado intencional sob certas condições; e, (2) um modo psicológico específico, que determina como o conteúdo intencional e as condições de satisfação se ajustam entre si (Searle, 1983).

Por exemplo, minha CRENÇA [de que está chovendo] tem como conteúdo intencional, [de que está chovendo], porque ele determina que as condições de satisfação desse estado intencional é que esteja de fato chovendo sob certos aspectos, e nenhuma outra condição. E como modo psicológico desse estado intencional, tem-se uma crença, que determina que esse conteúdo intencional se ajuste a essas condições de satisfação para que o estado intencional seja verdadeiro.

Por outro lado, meu DESEJO [de viajar para o Caribe] tem como conteúdo intencional, [de viajar para o Caribe], porque ele determina que que eu viaje de fato para o Caribe sob certos aspectos, sejam as condições de satisfação desse estado intencional; e um desejo, como modo psicológico desse estado Intencional, que determina que essas condições de satisfação se ajustem a esse conteúdo intencional para que o estado intencional seja satisfeito.

Os estados intencionais não funcionam isoladamente, mas compõem uma rede de Intencionalidade, de modo que as condições de satisfação de cada estado intencional são determinadas em relação a essa rede. E a rede de intencionalidade determina as condições de satisfação dos estados intencionais que a compõem em função de um background (capacidades, habilidades, hábitos, práticas, atitudes, suposições e pressuposições mentais etc.), que não são estados intencionais, mas fornecem os pré-requisitos para o funcionamento da rede de Intencionalidade. $\mathrm{O}$ background é estabelecido pela história filogenética e por um conjunto específico de relações sociais e físicas do indivíduo com pessoas e objetos ou estados de coisas do mundo (Searle, 1983, 1992, 2004).

Podemos sugerir algumas relações entre os conceitos apresentados nas propostas de Searle e de Tomasello. Por exemplo, os conceitos de Intencionalidade/intencional (a distinção parece ser meramente sintática, sendo Intencionalidade, o substantivo e intencional, o adjetivo) em Searle e o conceito de intencional em Tomasello se referem a uma propriedade de certas atividades dos organismos de ser dirigida para. O conceito de condições de satisfação em Searle e o conceito de objetivos em Tomasello se referem aos aspectos do mundo aos quais certas atividades dos organismos são dirigidas. Já o conceito de estados intencionais em Searle e o conceito de intencionalidade em Tomasello se referem a certas atividades dos organismos que possuem a propriedade de ser dirigida para.

Tomasello e Searle parecem concordar que a intenção é um tipo de atividade consciente e intencional, i.e., dirigida para. Searle $(1983,2004)$ propôs ainda que a ocorrência de uma ação intencional não necessita ter sido previamente planejada, pois ela já possui uma intenção-na-ação, cujas condições de satisfação são a realização de um movimento/ estado do agente. Tomasello parece sugerir algo parecido quando afirma que as habilidades de compreensão de ações intencionais se desenvolvem anteriormente às habilidades de compreensão de ações planejadas (Tomasello et al., 2005a).

Uma diferença entre o conceito de intenção em Tomasello e de intenção prévia em Searle é que este propõe que a intenção prévia é um estado intencional cujo conteúdo intencional é equivalente a uma proposição completa, ao invés de um sistema de representações cognitivas. Isso não significa dizer que a intenção prévia seja verbal, mas que seu conteúdo especifica que um "estado integrado de coisas", e não apenas um aspecto específico do mundo, sejam as condições de satisfação dessa intenção (Searle, 1983).

Finalmente, as propostas de Tomasello e de Searle criticam as concepções dualistas da cognição humana, ao considerarem fenômenos mentais e comportamentais como aspectos de uma mesma realidade natural e que os fenômenos mentais são um produto sócio-biológico da história evolutiva da espécie humana. Biológico, porque envolvem habilidades cognitivas e sócio-cognitivas comuns a diversas espécies animais, mas que assumiram características específicas na espécie humana. Sociais, porque essas habilidades interagem com e são modificadas por processos culturais. Nesse sentido, ambos os autores rejeitam uma natureza metafísica da intencionalidade e da mente.

\section{Daniel C. Dennett e a Teoria do Sistema Intencional}

Dennett (1971, 1983, 1985, 1987, 1988) desenvolveu uma análise da intencionalidade ao discutir os modos de funcionamento de sistemas orgânicos (p.ex., espécie huma- 
na) e inorgânicos (artefatos). Segundo ele, os sistemas são selecionados/programados de modo a se adaptarem da melhor forma possível ao seu ambiente, tendo desenvolvido ao longo de sua história, padrões de funcionamento objetivamente estáveis (i.e., que podem ser descritos por qualquer observador externo, em termos de ocorrências regulares e apropriadas, sob condições específicas). A diversidade e complexidade desses padrões seriam derivadas do tipo de configuração originalmente implementada nesses sistemas.

Devido às características dessa configuração, Dennett $(1971,1987)$ considerou que a interpretação dos padrões de funcionamento objetivamente estáveis, a partir de descrições da configuração física (interpretações físicas) ou do desempenho regular dos sistemas (interpretações funcionalistas), é inadequada e insuficiente. Uma estratégia mais pragmática para interpretar esses padrões é atribuir aos sistemas a capacidade de operar em um nível quase-ótimo de funcionamento (racionalidade), em uma situação específica, dado certos parâmetros, objetivos e informações (intencionalidade).

Dennett (1987) rejeitou a hipótese da intencionalidade enquanto uma propriedade intrínseca à mente ou uma estrutura cerebral causalmente relevante. Segundo ele, não há qualquer característica semântica intrínseca aos sistemas intencionais, exceto sua própria configuração original. A atribuição de intencionalidade aos sistemas intencionais como interpretação de seu funcionamento é, diz ele, um recurso pragmático.

Contudo, Dennett considerou que a intencionalidade não é uma mera ficção explanatória, porque padrões de funcionamento objetivamente estáveis são eventos reais e fenômenos cognitivos, como crenças, desejos e motivações, ainda que produtos de um discurso verbal, são circunstâncias e condições de satisfação relevantes para a regulação das atividades dos sistemas, devido às experiência prévias desses sistemas (Dennett, 1971).

O tratamento de Dennett ao conceito de intencionalidade parece fazer referência a disposições comportamentais integradas em um modo de interação dos sistemas, selecionadas por um programa de configuração original. Com isso, podemos sugerir que o conceito de intencionalidade em sua proposta é um conceito disposicional, uma vez que o funcionamento dos sistemas (ao serem direcionados para parâmetros, objetivos e informações do mundo) deve ser caracterizado em termos de ocorrências regulares e apropriadas, sob condições específicas. Conseqüentemente, essa interpretação do conceito de intencionalidade se contrapõe aos pressupostos mentalistas tradicionalmente atribuídos ao tratamento desse conceito (ver também Állan, 2007).

Podemos sugerir que os tratamentos do conceito de intencionalidade por Tomasello e por Dennett são compatíveis, na medida em que se referem a uma propriedade de certas habilidades cognitivas (Tomasello) ou do funcionamento dos sistemas intencionais (Dennett) de serem direcionados para objetivos. A principal diferença entre esses tratamentos é que Tomasello propõe que a intencionalidade é uma propriedade intrínseca a essas habilidades, enquanto que Dennett propõe que a atribuição de intencionalidade aos sistemas é uma atitude pragmática a posteriori.

Em comparação à hipótese de Tomasello de que a ação intencional é causada por uma intenção prévia envolvendo representações cognitivas, Dennett parece considerar que é um recurso pragmático interpretar que os sistemas planejem suas ações, antecipando possíveis resultados, e escolhendo suas ações com base no valor desses resultados, a partir de parâmetros disponíveis em seu contexto. Mas isso não envolveria qualquer tipo de representação mental desses parâmetros. Nesse sentido, os tratamentos do conceito de intenção por Dennett e por Tomasello parecem ser incompatíveis.

Em adição, podemos sugerir que tanto Tomasello quanto Dennett têm em comum a crítica às abordagens tradicionais da cognição humana, sob a justificativa de que essas abordagens não propõem um tratamento adequado da intencionalidade, segundo uma perspectiva evolucionista, que integre os níveis filogenético, ontogenético e cultural.

\section{Interpretações Funcionalistas da Intencionalidade}

As abordagens funcionalistas da cognição humana na psicologia comportamental não propuseram um tratamento específico do conceito de intencionalidade, limitando a análise desse conceito no contexto de um tratamento mais amplo dos conceitos "mentais". Basear-nos-emos nesse tratamento para sugerir uma interpretação do conceito de intencionalidade, segundo uma perspectiva funcionalista.

Com o conceito de operante, Skinner (1963, 1974/1976) integrou as noções de propósito e intenção à própria natureza funcional da relação entre o comportamento dos organismos e seu ambiente, no sentido de o que se segue ao comportamento e o modifica (ver também Day, 1975/1976). Segundo Skinner, o comportamento pode ser dito "direcionado" para algum propósito (ou intencional) quando suas conseqüências passadas o tornaram mais provável de ocorrer nessa direção do que em outra.

Day (1975/1976) defendeu e justificou a incorporação dos conceitos de intenção e intencionalidade ao escopo de investigação de uma ciência do comportamento, como sendo aspectos úteis para a interpretação de fenômenos comportamentais. Segundo ele, a intenção são as condições fisiológicas que controlam descrições verbais de estados psicológicos. No entanto, essas condições não possuem função causal, pois as variáveis de controle do comportamento são as condições ambientais descritas nas contingências de reforçamento. A intencionalidade, segundo Day, é uma característica intrínseca aos episódios comportamentais, relacionada aos efeitos do reforçamento sobre o comportamento operante. Portanto, ela pode ser considerada um conceito disposicional, em termos de uma tendência do comportamento operante de ser afetado de certo modo por condições subseqüentes a ele.

Rachlin (1992, 1994, 2003) propôs, com o conceito de padrões comportamentais temporalmente estendidos e ampliados, que os fenômenos mentais não são atividades de partes dos organismos, mas padrões de interação dos organismos como um todo, distribuídos temporalmente em contextos constituintes de um sistema social organizado. $\mathrm{O}$ comportamento pode ser dito "intencional" no sentido de que ele é realizado de modo a se "ajustar" a certo aspecto de um contexto que se destaca como a "causa final" mais relevante para o comportamento, em comparação com outros aspectos 
que, no mesmo contexto, podem também ser relacionados a esse comportamento (ver também Dutra, 2006). Portanto, é possível sugerir que o conceito de intencionalidade na proposta de Rachlin se refira a uma relação disposicional/ teleológica/externalista entre padrões comportamentais e contextos sociais.

Foxall (1999, 2004, 2007, 2008) propôs uma atualização da teoria do sistema intencional de Dennett, destacando a importância de descrições dos fenômenos comportamentais em termos intencionalistas, aliada a uma interpretação funcionalista. Segundo Foxall, a atribuição de intencionalidade aos organismos é uma estratégia heurística para prever e explicar seu comportamento complexo.

A diferença entre a proposta de Foxall, denominada behaviorismo intencional, e a proposta de Dennett, é que, ao invés de partir de correlatos neurofisiológicos da atividade cognitiva (nível sub-pessoal) para a atribuição de intencionalidade e racionalidade aos sistemas (nível pessoal), o behaviorismo intencionalista interpreta os fenômenos mentais a partir de observações de relações comportamentais (nível super-pessoal), que são confirmadas pela atribuição de intencionalidade e racionalidade aos organismos (nível pessoal), na medida em que elas fornecem evidências heurísticas para a pesquisa neurofisiológica (nível sub-pessoal), no âmbito de dados evolutivamente consistentes (para análises desta proposta, ver Baum, 2007; Branch, 2007; Burgos, 2007; Hocutt, 2007; Kileen, 2007; Lacey, 2007; Moore, 2007; Rachlin, 2007; Tonneau, 2007; para respostas a estas análises, ver Foxall, 2008).

Também Hineline (2003) e Neuman (2004, 2007) analisaram funcionalmente as circunstâncias nas quais o conceito de intenção é usado e atribuído como causa relevante para o comportamento. Essas circunstâncias envolvem geralmente condições internas dos indivíduos (Hineline, 2003) ou verbalizações em situações de controle instrucional (Neuman, 2004).

Neuman (2007) afirmou que, embora os conceitos de intenção e intencionalidade sejam funcionalmente distintos, as interpretações comportamentais não propõem definições operacionais específicas a cada um desses conceitos. Neuman definiu a intenção como verbalizações (comportamento verbal) que afetam outros comportamentos e a intencionalidade, como comportamentos modelados por contingências quando as conseqüências alternativas desses comportamentos são claras para aqueles que as observam e interpretam.

Considerando a interpretação que Állan (2007) propôs do tratamento skinneriano do conceito de intencionalidade como uma propriedade funcional do comportamento operante (ver também de Rose, 1982), é possível relacionar os tratamentos desse conceito por Skinner e por Tomasello, no sentido de uma propriedade de certos comportamentos (Skinner) ou certas habilidades (Tomasello) de "serem direcionado(a) s para" a conseqüência (Skinner) ou objetivo (Tomasello). Além disso, o tratamento de Tomasello do conceito de intencionalidade parece ser compatível com as interpretações de Day (1975/1976), Rachlin (1992, 1994, 2003) e Foxall (1999, 2004, 2007, 2008), do conceito de intencionalidade, em termos de uma disposição do comportamento de ser afetado por suas conseqüências.

Como dito anteriormente, o tratamento de Tomasello do conceito de intenção em termos de planejamento de ações intencionais, por meio de representações cognitivas, é incompatível com interpretações funcionalistas da cognição humana (p.ex., psicologia comportamental), por enfatizar pressupostos representacionalistas. No entanto, podemos sugerir como alternativa para conciliar esse conceito com pressupostos funcionalistas, considerar a intenção como comportamentos pré-correntes, i.e., comportamentos que maximizam a probabilidade de reforçamento de comportamentos subseqüentes (ver também Neuman, 2004, 2007).

Especificamente, segundo essa interpretação, a intenção são repertórios descritivos de contingências de reforçamento, estabelecidos por uma comunidade verbal (ver também Hineline, 2003), que aumentam a probabilidade de que os comportamentos subseqüentes a essas descrições verbais fiquem sob controle das condições especificadas nessas descrições. Por exemplo, minha intenção de abrir a porta é uma descrição de contingências de reforçamento, envolvendo condições antecedentes (p.ex., porta fechada) e conseqüentes (p.ex., porta aberta) a um curso de ação (p.ex., colocar a chave na fechadura da porta, girar a chave e a maçaneta, empurrar ou puxar a porta), sendo que esse último possui uma alta probabilidade de ocorrer, dada essas condições, devido tanto essas condições quanto esse curso de ação terem sido especificados nessas descrições verbais.

Também é possível sugerir uma relação entre os conceitos de estímulo reforçador (Skinner), de contexto social (Rachlin), e de objetivo (Tomasello), no sentido de que esses conceitos se referem a objetos ou estados de coisas do mundo, os quais a atividade humana é direcionada para (Állan, 2007). Obviamente, devemos fazer ressalvas quanto ao tratamento de Tomasello ao conceito de objetivo, na medida em que ele propõe em certos trabalhos que os objetivos não são objetos ou estados de coisas do mundo em si, mas representações cognitivas desses objetos ou estados de coisas. Esse tratamento torna o conceito de objetivo na abordagem de Tomasello incompatível com pressupostos funcionalistas nos quais os conceitos de estímulo reforçador e contexto social são fundamentados.

\section{Conclusão}

No presente trabalho, vimos que uma das principais críticas à abordagem de Tomasello da evolução da cognição humana é sua falta de elaboração e imprecisão conceitual, gerando ambigüidades e poucas informações sobre os processos envolvidos na evolução da cognição humana. Além disso, seu tratamento conceitual sugere uma interpretação mentalista da cognição humana.

Nesse sentido, buscamos analisar o tratamento de alguns dos principais conceitos apresentados na abordagem de Tomasello da evolução da cognição humana, em especial, o conceito de intencionalidade, na tentativa de esclarecer o papel desse conceito no contexto dessa abordagem. Buscando ainda estabelecer interlocuções da abordagem de Tomasello com outras abordagens do desenvolvimento cognitivo humano, comparamos o tratamento que Tomasello dá ao conceito de intencionalidade com o tratamento desse conceito nas teorias de Searle e de Dennett. Finalmente, buscando identificar 
compatibilidades da abordagem de Tomasello da evolução da cognição humana com pressupostos funcionalistas da cognição humana, comparamos o tratamento de Tomasello do conceito de intencionalidade com o tratamento desse conceito em abordagens funcionalistas da cognição humana na psicologia comportamental.

Interpretamos o conceito de intencionalidade na abordagem de Tomasello como uma propriedade de certas habilidades cognitivas de serem direcionadas para objetivos. Nesse sentido, ela é compatível com o tratamento do conceito de intencionalidade nas teorias de Searle - em termos de uma propriedade de certos estados ou processos mentais de serem acerca de objetos ou estados de coisas do mundo -, e de Dennett - em termos de uma propriedade do funcionamento dos sistemas orgânicos e inorgânicos, a partir de parâmetros, objetivos e informações disponíveis no mundo.

Obviamente, o tratamento de Tomasello e de Searle do conceito de intencionalidade se distingue do tratamento de Dennett desse conceito no que se refere à natureza da intencionalidade, a qual é considerada por Tomasello e por Searle uma propriedade intrínseca aos organismos, e considerada por Dennett uma propriedade derivada das práticas lingüísticas humanas.

Sugerimos também que é possível relacionar o conceito de intencionalidade na abordagem de Tomasello com interpretações funcionalistas e disposicionais da intencionalidade, como uma propriedade funcional da atividade dos organismos de ser afetável por condições e contextos subseqüentes. $\mathrm{O}$ conceito de motivação para compartilhar intencionalidade, na abordagem de Tomasello, pode também ser interpretado em termos disposicionais (Ryle, 1949/1969), na medida em que ele se refere a padrões ou tendências de ocorrências de certas atividades humanas, envolvendo o compartilhamento de objetivos comuns..

Por outro lado, o tratamento de Tomasello do conceito de objetivo é ambíguo porque alterna entre uma definição funcional - em termos de "causa final" - e uma definição mentalista - em termos de representação cognitiva de uma "causa final". No primeiro caso, o conceito de objetivo é compatível com os conceitos de condição de satisfação (Searle), de estímulo reforçador (Skinner, Day), e de contexto social (Rachlin).

O presente trabalho sugere que alguns conceitos utilizados na abordagem de Tomasello da evolução da cognição humana (intencionalidade, intencional e motivação) são compatíveis com conceitos aplicados em outras áreas do conhecimento, como a filosofia da mente e a psicologia comportamental. Assim, as críticas freqüentemente atribuídas à falta de elaboração e imprecisão conceitual na abordagem de Tomasello podem ser respondidas, respaldando-se nessas áreas.

Por outro lado, sugerimos que alguns conceitos utilizados na abordagem de Tomasello (objetivo e intenção) são problemáticos, na medida em que eles se aproximam de pressupostos mentalistas, e, por conseguinte, podem dificultar a interlocução com abordagens funcionalistas da cognição humana. No presente trabalho, tentamos oferecer algumas possibilidades de reformulação desses conceitos, por meio da adoção de definições funcionalistas e disposicionais.

\section{Referências}

Állan, S. (2007). Intencionalidade, linguagem e comportamento (Dissertação de mestrado não publicada). Universidade Federal do Pará, Belém.

Állan, S., \& Souza, C. B. A. (2009). O modelo de Tomasello sobre a evolução cognitivo-lingüístico humana. Psicologia: Teoria e Pesquisa, 25, 161-168.

Baum, W. M. (2007). Commentary on Foxall, "Intentional behaviorism." Behavior and Philosophy, 35, 57-60.

Branch, M. N. (2007). Are Foxall's intentions good? Behavior and Philosophy, 35, 61-64.

Burgos, J. E. (2007). About aboutness: Thoughts on intentional behaviorism. Behavior and Philosophy, 35, 65-76.

Day, W. F. (1976). Contemporary behaviorism and the concept of intention. In W. J. Arnold (Ed.), Nebraska Symposium on Motivation, 23 (pp. 55-131). London: University of Nebraska Press. (Trabalho original publicado em 1975)

Dennett, D. C. (1971). Intentional systems. Journal of Philosophy, 68, 87-106.

Dennett, D. C. (1983). Taking the intentional stance seriously: Author's response. Behavioral and Brain Sciences, 6, 379-388.

Dennett, D. C. (1985). When does the intentional stance work? Behavioral and Brain Sciences, 8, 763-766.

Dennett, D. C. (1987). The intentional stance. Cambridge, MA: MIT Press/Bradford Book.

Dennett, D. C. (1988). Précis of the intentional stance. Behavioral and Brain Sciences, 11, 495-546.

Dutra, L. H. de A. (2006). Comportamento intencional e contextos sociais: Uma abordagem nomológica. Abstracta, 2, 102-128.

Foxall, G. R. (1999). The contextual stance. Philosophical Psychology, 12, 21-42.

Foxall, G. R. (2004). Context and cognition: Interpretating complex behavior. Reno, NV: Context Press.

Foxall, G. R. (2007). Intentional behaviorism. Behavior and Philosophy, 35, 1-55.

Foxall, G. R. (2008). Intentional behaviorism revisited. Behavior and Philosophy, 36, 113-155.

Hineline, P. N. (2003). When we speak of intentions. In K. A. Lattal \& P. N. Chase (Eds.), Behavior Theory and Philosophy (pp. 203-221). New York: Kluwer Academic/Plenum Publishers.

Hocutt, M. (2007). Gordon Foxall on intentional behaviorism. Behavior and Philosophy, 35, 77-92.

Kileen, P. (2007). Fuzzy logic. Behavior and Philosophy, 35, 93-100.

Lacey, H. (2007). Intentional behaviorism and the intentional scheme: Comments on Gordon L. Foxall's "Intentional behaviorism" Behavior and Philosophy, 35, 101-111.

Moore, J. (2007). Comments on "Intentional Behaviorism" by G. R. Foxall. Behavior and Philosophy, 35, 113-130.

Neuman, P. (2004). An intentional interpretative perspective. The Behavior Analyst, 27, 55-65.

Neuman, P. (2007). Some comments on the distinction between intention and intentionality. The Behavior Analyst, 30, 211-216.

Rachlin, H. (1992). Teleological behaviorism. American Psychologist, 47, 1371-1382.

Rachlin, H. (1994). Behavior and mind: The roots of modern psychology. Oxford University Press.

Rachlin, H. (2003). Privacy. In K. A. Lattal \& P. N. Chase (Eds.), Behavior theory and philosophy (pp. 187-201). New York: Kluwer Academic/Plenum Publishers. 
Rachlin, H. (2007). A Behavioral science of mental life: Comments on Foxall's "Intentional behaviorism." Behavior and Philosophy, 35, 131-138.

Rose, J. C. C. de (1982). Consciência e propósito no behaviorismo radical. In B. Prado Jr. (Ed.), Filosofia e Comportamento (pp. 67- 91). São Paulo: Brasiliense.

Ryle, G. (1969). The concept of mind. London: Hutchinson of London. (Trabalho original publicado em 1949)

Searle, J. R. (1983). Intentionality. Cambridge University Press.

Searle, J. R. (1990a). Consciousness, explanatory inversion and cognitive science. Behavioral and Brain Sciences, 13, 585-596.

Searle, J. R. (1990b). Who is computing with the brain? Behavioral and Brain Sciences, 13, 632-640.

Searle, J. R. (1992). The rediscovery of the mind. Cambridge, MA: The MIT Press.

Searle, J. R. (1997). Consciousness denied: Daniel Dennett's account. In The mystery of consciousness (pp. 96-131). New York Review Book.

Searle, J. R. (1998). How the mind works: Intentionality. In J. R. Searle (Ed.). Mind, language, and society: Philosophy in the real world (pp. 85-109). New York: Basic Books.

Searle, J. R. (2004). Mind: A brief introduction. Oxford University Press.

Skinner, B. F. (1963). Operant behavior. American Psychologist, $18,503-515$.
Skinner, B. F. (1976). About behaviorism. New York: Vintage Books. Trabalho original publicado em 1974.

Tomasello, M. (1999). The cultural origins of human cognition. Cambridge, MA/London: Harvard University Press.

Tomasello, M. (2003). Constructing a language: A usage-based theory of language acquisition. Cambridge, MA/London: Harvard University Press.

Tomasello, M., Carpenter, M., Call, J., Behne, T., \& Moll, H. (2005a). Understanding and sharing intentions: The origins of cultural cognition. Behavioral and Brain Sciences, 28, 675-691.

Tomasello, M., Carpenter, M., Call, J., Behne, T., \& Moll, H. (2005b). In search of the uniquely human. Behavioral and Brain Sciences, 28, 721-727.

Tonneau, F. (2007). Behaviorism and Chisholm's challenge. Behavior and Philosophy, 35, 139-148.

Recebido em 09.10.2009

Primeira decisão editorial em 12.05.2010

Versão final em 11.06.2010

Aceito em 12.08.2010

\section{CONGRESSO INTERDISCIPLINAR DE DOR DA USP}

Período: 16 de Junho - 18 Junho 2011

Local: São Paulo-SP, BR 